\title{
THE STRUCTURE OF THE UNIVERSAL EXPONENTIAL SOLUTION OF THE YANG-BAXTER EQUATION
}

\author{
NANTEL Bergeron
}

\begin{abstract}
A в stract. S. Fomin and A. Kirillov have shown that exponential solutions of the Yang-Baxter equation give rise to generalized Schubert polynomials and corresponding symmetric functions, and they provided several equivalent descriptions of the local stationary algebra $\mathcal{A}_{0}$ defined by this equation. Here we show that $\mathcal{A}_{0}$ is isomorphic to the graded associative algebra formally generated by the elements $a, C_{0}, C_{1}, C_{2}, \ldots$ satisfying the relations $\left[a, C_{i}\right]=C_{i+1}$ and $\left[C_{i}, C_{j}\right]=0$. The rank of $C_{i}$ is $i+1$. It will follow that the Hilbert series of $\mathcal{A}_{0}$ is $\frac{1}{(1-t)^{2}} \frac{1}{1-t^{2}} \frac{1}{1-t^{3}} \cdots$.
\end{abstract}

\section{Introduction}

We will follow the treatment of S. Fomin and A. Kirillov [2] to introduce our problem. Let $\mathbb{K}$ be a field of characteristic zero. Let $\mathcal{A}=$ $\mathbb{K}\left[u_{1}, u_{2}, \ldots\right](x, y, \ldots)$ be the associative algebra of formal power series in commuting variables $x, y, \ldots$ with coefficients in a local stationary algebra [9] with generators $u_{1}, u_{2}, \ldots$ That is we assume $u_{i}, u_{j}$ commute if $|i-j|>1$ and $u_{i}, u_{i+1}$ are subject to certain relations which are invariant in $i$.

It was shown $[1,4]$ that a theory of generalized Schubert polynomials and corresponding Stanley's symmetric functions can be developed whenever one has a solution of the Yang-Baxter equation

$$
h_{i}(x) h_{i+1}(x+y) h_{i}(y)=h_{i+1}(y) h_{i}(x+y) h_{i+1}(x)
$$

where $h_{i}(x)=e^{x u_{i}}$. That is

$$
e^{x a} e^{(x+y) b} e^{y a}=e^{y b} e^{(x+y) a} e^{x b}
$$

holds for any pair of adjacent generators $a=u_{i}$ and $b=u_{i+1}$.

Received September 10, 1993.

Work supported by NSF grant. 
The second section of this paper is devoted to the Theorem of S. Fomin and A. Kirillov [2] which gives a minimal set of relations on $a, b$ that guarantee (1.1). In other words, they characterize the local stationary algebra $\mathcal{A}_{0}$ defined by (1.1). In the third section we recall some of the theory of free Lie algebras and Lyndon words $[5,7,8,10]$. This will be needed in the fourth section where we show that $\mathcal{A}_{0}$ is isomorphic to the graded associative algebra formally generated by the elements $a, C_{0}, C_{1}, C_{2}, \ldots$ satisfying the relations $\left[a, C_{i}\right]=C_{i+1}$ and $\left[C_{i}, C_{j}\right]=0$. The rank of $C_{i}$ is $i+1$. It will follow that the Hilbert series of $\mathcal{A}_{0}$ is

$$
\frac{1}{(1-t)^{2}} \frac{1}{1-t^{2}} \frac{1}{1-t^{3}} \cdots
$$

\section{Fomin and Kirillov's results}

We are interested in the graded associative algebra $\mathcal{A}_{0}$ formally generated by the elements $a, b$ satisfying the relations implied by (1.1). That is, the coefficients of $x^{m} y^{n}$ on both sides of (1.1) impose (infinitely many) relations on $a, b$. Any other exponential solution of (1.1) can be represented as a quotient of $\mathcal{A}_{0}$ by a certain ideal. It is in this sense that $\mathcal{A}_{0}$ is universal.

The main result of [2] is to extract out of (1.1) equivalent minimal sets of relations. To state it, we need some notations. Let $C_{0}=C_{0}(a, b)=a+b$ and $C_{i}=C_{i}(a, b)=\left[a, C_{i-1}\right]$ for $i \geq 1$. Here $[-,-]$ stand for the Lie bracket $[f, g]=f g-g f$.

Theorem 2.1 [2]. $\mathcal{A}_{0}$ is the graded associative algebra formally generated by $a, b$ satisfying the relations $\left[C_{i}(a, b), C_{j}(a, b)\right]=0$ for all $i, j \geq 0$.

Remark 2.2. In [2] the reader will find many other equivalent minimal sets of relations. The one in Theorem 2.1 suits us best.

Although Theorem 2.1 characterizes the algebra $\mathcal{A}_{0}$ it is not completely satisfactory. For instance it does not provide the dimension of each homogeneous part of $\mathcal{A}_{0}$ (Hilbert series). To better understand $\mathcal{A}_{0}$, one must seek an even more precise description. This will be the purpose of Section 4. But before we get into this, let us repeat here the examples of solution of (1.1) given in [2]. This is what motivates the study of the universal algebra $\mathcal{A}_{0}$.

Example 2.3 (Hecke Algebra). Fix $\beta \in \mathbb{K}$. Consider the local algebra with generator $u_{i}$ satisfying (1.1) and

$$
u_{i}^{2}=\beta u_{i}
$$


That is $a^{2}=\beta a$ and $b^{2}=\beta b$ in our algebra $\mathcal{A}_{0}$. The relation $\left[C_{0}, C_{1}\right]=0$ implies that $a b a=b a b$ (Coxeter relation). Also recall that our local algebras must satisfy $u_{i} u_{j}=u_{j} u_{i}$ for $|i-j|>1$. We recognize here the relations of a Hecke algebra. In other words, a local algebra satisfying (1.1) and (2.1) is a Hecke algebra.

Example 2.4 (Schubert and Grothendiek Polynomials). Specializing Example 2.3 to $\beta=0$ and $\beta=1$, one can construct the Schubert and Grothendiek Polynomials of Lascoux and Schützenberger [6] and also some related symmetric polynomials (see $[1,3,4]$ ).

Example 2.5 (Universal Enveloping Algebra $U_{+}(\mathfrak{g l}(n))$ and the Local Heisenberg Algebra). $U_{+}(\mathfrak{g l}(n))$ is generated by $u_{1}, u_{2}, \ldots$ subject to the Serre relations

$$
\left[u_{i},\left[u_{i}, u_{i \pm 1}\right]\right]=0
$$

That is, $[a,[a, b]]=[b,[b, a]]=0$. This implies $\left[C_{0}, C_{1}\right]=0$ and $C_{2}=0$. Hence $U_{+}(\mathfrak{g l}(n))$ is a local algebra satisfying (1.1). If we impose the even stronger relation $[a, b]=\lambda$, we get the local Heisenberg algebra.

\section{Free Lie algebras and Lyndon words}

Before we state and prove our main Theorem, we need to recall some of the theory of free Lie algebras. The reader can find various treatments of this in many sources $[5,7,8,10]$.

Let $\mathfrak{A}=\{a, b\}$ be an alphabet consisting of two ordered letters $a<b$. Let $\mathfrak{A}^{*}$ be the free monoid generated by $\mathfrak{A}$. We can view $\mathfrak{A}^{*}$ as the set of all words in the alphabet $\mathfrak{A}$, equipped with the concatenation product and a neutral element denoted 1, the empty word. For $w \in \mathfrak{A}^{*}$, we denote by $|w|$ the length of $w$, this is a monoid homomorphism $\mathfrak{A}^{*} \rightarrow \mathbb{Z}^{+}$. The lexicographic order of words form a total order on $\mathfrak{A}^{*}$.

Let $\mathbb{K} \mathfrak{A}$ be the free associative algebra generated by $\mathfrak{A}$. The elements of $\mathbb{K} \mathfrak{A}$ are sums of the form

$$
\sum_{w \in \mathfrak{A}^{*}} c_{w} w
$$

with finite support and $c_{w} \in \mathbb{K}$. The product in $\mathbb{K} \mathfrak{A}$ is the linearization of the product in $\mathfrak{A}^{*}$. This algebra is naturally graded by the length function $1-1$. 
Now we consider $\operatorname{Lie}(\mathfrak{A}) \subset \mathbb{K} \mathfrak{A}$ the (free) Lie algebra generated by $\mathfrak{A}$ with the Lie bracket $[f, g]=f g-g f$. Recall that the Lie bracket satisfies the Jacobi identity which can be expressed as follows:

$$
[[f, g], h]=[f,[g, h]]+[[f, h], g] .
$$

In this setting the Poincaré-Birkhoff-Witt Theorem states (see [5,7]), that the universal enveloping algebra $U(\operatorname{Lie}(\mathfrak{A}))$ is exactly $\mathbb{K} \mathfrak{A}$.

The next step is to construct a linear basis of $\operatorname{Lie}(\mathfrak{A})$. For this, we introduce Lyndon words. A word $\ell \in \mathfrak{A}^{*}$ is called Lyndon if it is strictly smaller (lexicographically) than all of its non-trivial circular rearrangements.

Example 3.1. $a, b, a b, a a b, a b b, a a b b, a a b a b, \ldots$ are all Lyndon words, but $a a, a b a b, a b a, a b a a b, \ldots$ are not.

Here are some standard propositions on Lyndon words $[5,7,8,10]$.

Proposition 3.2. If $\ell_{1}, \ell_{2} \in \mathfrak{A}^{*}$ are both Lyndon words such that $\ell_{1}<\ell_{2}$, then the word $\ell_{1} \ell_{2}$ is Lyndon.

Proposition 3.3. Let $\ell \in \mathfrak{A}^{*}$ be a Lyndon word of length $\geq 2$. Let $\ell=u \ell_{2}$ be the unique factorization of $\ell$ such that $u \neq 1$ and $\ell_{2}$ is the longest possible Lyndon word. Then $u$ is also a Lyndon word.

Proposition 3.4. Given $w \in \mathfrak{A}^{*}$ there exists a unique factorization of $w$ into Lyndon words $w=\ell_{1} \ell_{2} \cdots \ell_{k}$ such that $\ell_{1} \geq \ell_{2} \geq \cdots \geq \ell_{k}$.

Using Proposition 3.3 we define recursively the standard bracketing $[\ell]$ of a Lyndon word $\ell \in \mathfrak{A}^{*}$ as follows. If $|\ell|=1$ then set

$$
[\ell]=\ell \text {, }
$$

if $|\ell| \geq 2$ Proposition 3.3 gives us a standard factorization into two Lyndon words $\ell=\ell_{1} \ell_{2}$, and we set (recursively)

$$
[\ell]=\left[\left[\ell_{1}\right],\left[\ell_{2}\right]\right] \text {. }
$$

For example, if $n \geq 1$ then $\ell=a^{n} b$ is Lyndon and $[\ell]=C_{n}=\left[a,\left[a^{n-1} b\right]\right]$. With this in hand, we have

Proposition 3.5. A linear basis of Lie $(\mathfrak{A})$ is given by

$$
\left\{[\ell]: \ell \in \mathfrak{A}^{*} \text { is Lyndon }\right\} \text {. }
$$

Proposition 3.6 [Poincaré-Birkhoff-Witt]. A linear basis of $\mathbb{K} \mathfrak{A}=$ $U(\operatorname{Lie}(\mathfrak{A}))$ is given by

$$
\left\{\left[\ell_{1}\right]\left[\ell_{2}\right] \cdots\left[\ell_{k}\right]: \ell_{i} \in \mathfrak{A}^{*} \text { is Lyndon and } \ell_{1} \geq \ell_{2} \geq \cdots \geq \ell_{k}\right\} .
$$




\section{The algebra $\mathcal{A}_{0}$}

We are now in a position to improve the description of the algebra $\mathcal{A}_{0}$. Let $\mathfrak{I}=\left\langle\left[C_{i}, C_{j}\right]: i, j \geq 0\right\rangle \subset \mathbb{K} \mathfrak{A}$ be the ideal generated by the relations of Theorem 2.1. We have

$$
\mathcal{A}_{0}=\mathbb{K} \mathfrak{A} / \mathfrak{I}
$$

From Proposition 3.6 we have that $\mathbb{K} \mathfrak{A}=U(\operatorname{Lie}(\mathfrak{A}))$. Let $L(\mathfrak{I})=\left\langle\left[C_{i}, C_{j}\right]\right.$ : $i, j \geq 0\rangle \subset \operatorname{Lie}(\mathfrak{A})$ be the Lie ideal generated by the relations of Theorem 2.1. This makes sense since $a, C_{0}, C_{1}, C_{2}, \ldots$ and $\left[C_{i}, C_{j}\right]$ all belong to $\operatorname{Lie}(\mathfrak{A})$. On the other hand we have that $L(\mathfrak{I}) \subset \mathfrak{I}$. Hence we can view (4.1) as follows:

$$
\mathcal{A}_{0}=U(\operatorname{Lie}(\mathfrak{A})) / \mathfrak{I}=U(\operatorname{Lie}(\mathfrak{A}) / L(\mathfrak{I}))
$$

Our main Theorem will be to describe the Lie algebra appearing in the second equality of (4.2). For this, let

$$
L\left(\mathcal{A}_{0}\right)=\operatorname{Lie}(\mathfrak{A}) / L(\mathfrak{I})
$$

Theorem 4.1. $\left\{a, C_{0}, C_{1}, C_{2}, \ldots\right\}$ is a linear basis of $L\left(\mathcal{A}_{0}\right)$.

Proof. From Theorem 3.5, we know that $\left\{[\ell]: \ell \in \mathfrak{A}^{*}\right.$ is Lyndon $\}$ spans $L\left(\mathcal{A}_{0}\right)$. We will show that modulo the relations in $L(\mathfrak{I})$, with $\ell \in \mathfrak{A}^{*}$ Lyndon, we have

$$
[\ell] \equiv \begin{cases}C_{m-1}=\left[a^{m-1} b\right] & \text { if } \ell=a^{n} b^{m-n}, n \geq 1 \text { and } m>n \\ \ell & \text { if }|\ell|=1 \\ 0 & \text { otherwise }\end{cases}
$$

This in turn will show our claim since the set $\left\{a, C_{0}, C_{1}, C_{2}, \ldots\right\}$ is clearly independent in $L\left(\mathcal{A}_{0}\right)$.

We proceed by induction on $m=|\ell|$. If $|\ell|=1$ then either $[\ell]=a$ or $[\ell]=b=C_{0}-a$. If $|\ell|=2$ then $[\ell]=[a, b]=C_{1}$. Now suppose that

$|\ell|=m>2$. Assume that (4.4) holds for all $\left|\ell^{\prime}\right|<m$. We will consider two cases. 
Case I. $\ell=a^{n} b^{m-n}$ : Notice that we must have $0<n<m$. Suppose first that $n>1$. Then

$$
\begin{aligned}
(3.2) & \Rightarrow & {[\ell] } & =\left[a^{n} b^{m-n}\right]=\left[a,\left[a^{n-1} b^{m-n}\right]\right] \\
(4.4) & \Rightarrow & & \equiv\left[a,\left[a^{m-2} b\right]\right] \\
(3.2) & \Rightarrow & & =\left[a^{m-1} b\right]=C_{m-1} .
\end{aligned}
$$

Suppose next that $\ell=a b b$. The relation $\left[C_{0}, C_{1}\right]=0$ implies that $[a b b] \equiv$ $[a a b]=C_{2}$. Finally, assume that $n=1$ and $m>3$. By the induction hypothesis (4.4), we have that

$$
\left[a b^{m-2}\right] \equiv C_{m-2} \equiv\left[a a b^{m-3}\right] .
$$

Hence

$$
\begin{array}{rlrl}
(3.2) \Rightarrow & {[\ell]} & =\left[a b^{m-1}\right]=\left[\left[a b^{m-2}\right], b\right] \\
(4.5) \Rightarrow & & \equiv\left[\left[a a b^{m-3}\right], b\right] \\
(3.2) \Rightarrow & & =\left[\left[a,\left[a b^{m-3}\right]\right], b\right] \\
(3.1) \Rightarrow & & =\left[a,\left[\left[a b^{m-3}\right], b\right]+\left[[a, b],\left[a b^{m-3}\right]\right]\right. \\
(3.2) \Rightarrow & & =\left[a,\left[a b^{m-2}\right]\right]+\left[[a, b],\left[a b^{m-3}\right]\right] \\
(4.4) \Rightarrow & & \equiv\left[a,\left[a^{m-2} b\right]\right]+\left[C_{1}, C_{m-3}\right] \\
& & & \equiv C_{m-1}+0 .
\end{array}
$$

Case II. $\ell=u b a v$, where $u, v \in \mathfrak{A}^{*}$ : Let $\ell=\ell_{1} \ell_{2}$ be the standard factorization of Proposition 3.3. Assume first that $\ell_{1}=u^{\prime} b a v^{\prime}$, then by the induction hypothesis, we have $\left[\ell_{1}\right] \equiv 0$. Hence $[\ell]=\left[\left[\ell_{1}\right],\left[\ell_{2}\right]\right] \equiv 0$. Similarly, if $\ell_{2}=u^{\prime} b a v^{\prime}$ then $[\ell] \equiv 0$. Finally, we must assume that $\ell_{1}=a^{p} b^{q-p}$ and $\ell_{2}=a^{r} b^{s-r}$, where $0<p<q$ and $0<r<s$. Then $\left[\ell_{1}\right] \equiv C_{q-1}$, $\left[\ell_{2}\right]=C_{s-1}$ and $[\ell]=\left[\left[\ell_{1}\right],\left[\ell_{2}\right]\right] \equiv\left[C_{q-1}, C_{s-1}\right] \equiv 0$.

Now that we have a linear basis of the Lie algebra $L\left(\mathcal{A}_{0}\right)$, the PoincaréBirkhoff-Witt Theorem gives us the following corollary.

Corollary 4.2. A linear basis of $\mathcal{A}_{0}$ is given by

$$
\left\{a^{p} C_{0}^{q_{0}} C_{1}^{q_{1}} \cdots: \sum_{i \geq 0} q_{i}<\infty\right\} .
$$

The rank of $C_{i}$ is $i+1$, hence the rank of $a^{p} C_{0}^{q_{0}} C_{1}^{q_{1}} \cdots$ is $p+\sum q_{i}(i+1)$. This directly implies the following:

Corollary 4.3. The number of basis elements of Corollary 4.2 having rank $n$ is the coefficient of $t^{n}$ in the following Hilbert series

$$
\frac{1}{(1-t)^{2}} \frac{1}{1-t^{2}} \frac{1}{1-t^{3}} \frac{1}{1-t^{4}} \cdots
$$




\section{Acknowledgement}

The author is grateful to S. Fomin for sharing this beautiful mathematics.

\section{References}

1. S. Fomin and A. N. Kirillov, The Yang-Baxter equation, symmetric functions, and schubert polynomials, Proceedings of the 5th conf. on Formal Power Series and Algebraic Combinatorics, Firenze, 1993.

2. Universal exponential solution of the Yang-Baxter equation, preprint, MIT, 1993.

3. - Grothendieck polynomials and the Yang-Baxter equation, preprint, MIT, 1993.

4. S. Fomin and R. P. Stanley, Schubert polynomials and the NilCoxeter algebra, Adv. Math. (to appear).

5. A. Garsia, Combinatorics of the Free Lie Algebra and the Symmetric Group, Analysis, Et Cetera, Research papers published in honor of Jurgen Moser's 60th birthday (Paul H. Rabinowitz and Eduard Zehnder, eds.), Academic Press, 1990.

6. A. Lascoux, Polynômes de Schubert; une approche historique, Publication LACIM, Séries formelles et combinatoire algébrique 11 (1992), UQAM, Montréal, 283-296.

7. M. Lothaire, Combinatorics on words, Encyclopedia of Math., vol. 17, AddisonWesley, 1983.

8. C. Reutenauer, Free Lie Algebras, Oxford University press, 1993.

9. A. M. Vershik, Local Stationary Algebras, Amer. Math. Soc. Transl. (2), vol. 148, 1991, pp. 1-13.

10. X. G. Viennot, Algèbres de Lie libres et monö̈des libres, Lec. Notes in Math., vol. 691, Springer-Verlag, 1978.

Harvard University, Department of Mathematics, Cambidge, M a 02138

E-mail address: nantel@math.harvard.edu 